\title{
TRANSIÇÃO E ADAPTAÇÃO À UNIVERSIDADE Apresentação de um Questionário de Vivências Académicas (QVA)
}

\author{
Leandro S. Almeida, Ana Paula C. Soares e Joaquim Armando G. Ferreira
}

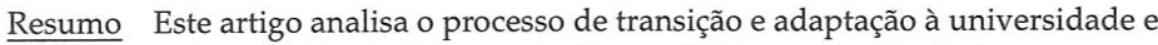
apresenta o Questionário de Vivências Académicas (QVA - Almeida \& Ferreira, 1997). Este questionário, enquanto instrumento de despiste, conceptualiza o processo de transição e adaptação à universidade como um processo complexo onde, tanto as variáveis pessoais como as variáveis académicas e contextuais, interagem, afectando o modo como os estudantes se adaptam, realizam e desenvolvem na instituição do Ensino Superior em que estão inseridos. O QVA foi administrado a uma amostra de 1273 alunos a frequentar o primeiro ano de 41 licenciaturas da Universidade do Minho. Os resultados nas 17 subescalas que compõem o QVA, demonstram boas qualidades psicométricas, quer no que se refere aos valores de consistência interna, quer à dimensionalidade, o que corrobora a sua utilização enquanto um instrumento de despiste.

Palavras-chave Ensino Superior; transição; adaptação; rendimento académico; desenvolvimento; vivências académicas.

\section{Transição e adaptação à universidade}

A transição do Ensino Secundário para o Ensino Superior, confronta os jovens com múltiplos desafios. ${ }^{1}$ A par das mudanças desenvolvimentais operadas numa fase final da adolescência e início da vida adulta, os estudantes vêem-se ainda confrontados com uma série de novos e complexos desafios, que decorrem quer das exigências que o novo contexto educativo lhes coloca, quer das implicações que esta transição poderá acarretar nos vários domínios da sua existência. A investigação sugere que esta transição confronta o jovem com a resolução simultânea de um conjunto de tarefas em quatro domínios principais: (i) académico (o ensino universitário requer novos ritmos e estratégias de aprendizagem, face aos novos sistemas de ensino e de avaliação); (ii) social (a experiência universitária requer o desenvolvimento de padrões de relacionamento interpessoal mais maduros na relação com a família, com os professores e os colegas, com o sexo oposto e com as figuras de autoridade); (iii) pessoal (os anos universitários devem concorrer para o estabelecimento de um forte sentido de identidade, para o desenvolvimento da auto-estima, de um maior conhecimento de si próprio/a e para o desenvolvimento de uma 
visão pessoal do mundo); e (iv) vocacional/institucional (a universidade constitui uma etapa fundamental para o desenvolvimento de uma identidade vocacional, onde a especificação, a implementação e o comprometimento com determinados objectivos vocacionais e/ou institucionais parecem assumir particular importância) (Baker, McNeil \& Siryk, 1985; Creamer, 1990; Evans, Forney \& Dibrito, 1998; Gonçalves \& Cruz, 1988; Gordan, 1995; Komives, Delworth \& Woodard, 1996; Scwitzer, Ancis \& Griffin, 1998; Soares, 1998; Upcraft \& Gardner, 1989).

Neste contexto, o primeiro ano da universidade tem sido conceptualizado como um período crítico, potencializador de crises e/ou desafios desenvolvimentais e como o principal determinante dos padrões de desenvolvimento estabelecidos pelos jovens ao longo da sua frequência universitária (Bastos, 1998; Bastos \& Gonçalves, 1996; Heath, 1968; Pascarella \& Terenzini, 1991). Por outro lado, partindo de resultados que sugerem que mais de metade dos estudantes que ingressam no Ensino Superior revelam dificuldades nesta transição educativa (Bauer \& Mott, 1990; Healy \& Reilly, 1989; Kramer, Berger \& Miller, 1974; cit. por Herr \& Cramer, 1992; Leitão \& Paixão, 1999), e de outros que sugerem mesmo um aumento de níveis de psicopatologia na população universitária (Ratingan, 1989; Stone \& Archer, 1990), os estudantes universitários em geral, e os que ingressam pela primeira vez no Ensino Superior em particular, têm-se assumido como uma população-chave no estudo dos processos de adaptação e desenvolvimento humanos em contexto universitário, tanto no plano internacional da investigação (Astin, 1975, 1984, 1993; Pace, 1984; Pascarella \& Terenzini, 1991; Russell \& Petrie, 1992; Tinto, 1987; Upcraft \& Gardner, 1989; Weidman, 1989), como, mais recentemente, no plano nacional (Bastos, 1998; Belo, 1999; Bento \& Ferreira, 1997; Carneiro, 1999; Costa, 1991; Costa \& Campos, 1986; Diniz \& Almeida, 1997; Faria \& Santos, 1998; Ferreira \& Castro, 1994; Ferreira \& Hood, 1990; Hood \& Ferreira, 1983; Marques \& Miranda, 1993; Martins, 1998; Medeiros, Ferreira \& Ponciano, 1997; Menezes, Costa \& Campos, 1989; Miranda, 1994; Nico, 1995; Soares, 1998; Tavares, Santiago, Lencastre \& Soares, 1996).

Estes estudos defendem, de um modo geral, que o processo de transição e adaptação/ajustamento ao contexto universitário deve ser conceptualizado como um processo complexo e multidimensional, envolvendo múltiplos factores tanto de natureza intra e interpessoal como de natureza contextual. Aliás, e de acordo com as perspectivas mais transacionais no estudo do desenvolvimento humano (Lowenthal \& Pierce, 1975; Neugarten, Moore \& Lowe, 1965; Pearlin, 1982; Schlossberg, 1981, 1998), para compreendermos a forma como os indivíduos lidam com os momentos de transiçãoe os resultados a que eles podem conduzir, devemos considerar quer as características pessoais que o indivíduo "traz" para o novo contexto, e que obrigatoriamente irão modelar a forma como o indivíduo o percepciona e nele actua, quer as características do próprio contexto para onde o indivíduo realiza a transição. ${ }^{2}$

Desta forma, e tendo em vista a apresentação do Questionário de Vivências Académicas - QVA (Almeida \& Ferreira, 1997), descrevemos três grupos de variáveis que nos parecem importantes na análise do processo de transição e adaptação dos estudantes ao Ensino Superior: (i) variáveis mais directamente associadas à 
aprendizagem e ao rendimento académico; (ii) variáveis mais directamente associadas ao self e ao desenvolvimento psicossocial dos indivíduos; e (iii) variáveis mais directamente associadas ao contexto universitário, as quais podem inclusive extravasar a própria instituição ou o espaço físico do campus.

\section{Aprendizagem e rendimento académico}

A questão do rendimento académico, na sua faceta mais visível do sucesso/insucesso, não tem merecido ainda amplo debate no seio da "academia". A meados de 1999 , as universidades portuguesas foram convidadas a elaborar contratos-programa para os próximos cinco anos (Despacho n. ${ }^{\circ} 6659 / 99$ ), tendo em vista a superação dos casos de insucesso persistente (alunos em situação de não elegibilidade para efeitos de financiamento - Lei 113/97). Por diferentes razões esta iniciativa suscitou surpresa nalguns académicos. De facto, fala-se habitualmente, e muito, do insucesso na Escolaridade Básica, bastante do insucesso no Ensino Secundário, mas tem-se "poupado" o Ensino Superior, nesta questão. No entanto, a massificação ocorrida no acesso e frequência do Ensino Superior nos vários países e em Portugal a partir da década de 70 (Arroteia, 1996; Braga da Cruz et al., 1995; Conceição et al., 1998; Education Trainning Youth, 1995; Gago et al., 1994), tem justificado alguns estudos e uma preocupação crescente face ao não menos volumoso índice de insucesso académico (baixas classificações, absentismo, diminuição nos critérios para a transição de ano, disciplinas em atraso, mudanças de curso, abandonos). A "democratização do Ensino Superior" conduziu efectivamente, como refere Braga da Cruz e colaboradores (1995), ao alargamento da base social de recrutamento estudantil, o que se tem repercutido, indubitavelmente, em algumas dimensões do sucesso académico.

Múltiplas variáveis aparecem associadas à aprendizagem e ao rendimento académico dos estudantes no Ensino Superior. Muito por paralelo com o que ocorre nos demais níveis de ensino, tais variáveis reportam-se ao próprio aluno, ao professor, à organização curricular, ao contexto académico e às múltiplas formas de interacção daí decorrentes. O peso de cada um destes grupos de variáveis não é totalmente autónomo dos demais, pelo menos quando se trata de implementar medidas preventivas ou remediativas de forma a minorar os respectivos impactos. Por outro lado, podemos defender que esse mesmo impacto depende do ano curricular em que o estudante se encontra, sendo lógico ser esse impacto maior junto dos alunos do primeiro ano, por vivenciarem uma fase de transição e de adaptação, com implicações aos vários níveis da sua existência.

Habitualmente associaram-se elevadas expectativas aos alunos que chegam à universidade, quer decorrentes de uma lógica de universidade elitista do passado, quer de uma prática selectiva instalada no presente e associada à política de $n u-$ merus clausus. Nem sempre, no entanto, tais expectativas são realistas e favoráveis à integração destes alunos no Ensino Superior, e à sua aprendizagem. Nesta altura, 
pode-se criticar o sistema e os professores anteriores que não souberam preparar adequadamente estes estudantes (atitude frequente nos professores dos sucessivos níveis de escolaridade), ou assumir que sem alunos não existe Ensino Superior e, desde logo, importa operacionalizar os problemas, vislumbrar e implementar as soluções possíveis. Como nos demais ciclos de escolaridade prévios à universidade, importa estreitar o diálogo e a cooperação com os subsistemas de escolarização a seu montante, sobretudo se se reconhece que algo pode já não ser tão facilmente conseguido, ou melhor conseguido, uma vez entrado no Ensino Superior.

A política de numerus clausus no acesso ao Ensino Superior merece, ainda, nova referência. Esta política está na origem de altas percentagens de alunos colocados em pares "estabelecimento de ensino/ curso" que não correspondem à sua primeira escolha. A título de exemplo, no início da década de 90 , apenas $32,4 \%$ dos estudantes do Ensino Universitário foram colocados na sua primeira opção (cf. Braga da Cruz et al., 1995). Mesmo que nos últimos anos a percentagem de jovens colocados no curso/estabelecimento preferido tenha vindo a aumentar (de referir que, na amostra deste artigo, 59\% dos alunos estão colocados no par curso/estabelecimento de ensino correspondente à sua primeira opção), é de considerar ainda o facto de muitos deles "manipularem" as suas opções na candidatura ao Ensino Superior, de forma a aumentar as suas probabilidades de ingresso neste nível de ensino (cf. Braga da Cruz et al., 1995). Desta forma, importa não atender apenas ao facto de o jovem ter conseguido ou não entrar na sua primeira opção, mas se esta opção correspondia verdadeiramente aos seus principais objectivos e intenções. Daqui decorrem, obviamente, formas diversas de estar na universidade e também níveis diferentes de investimento no estudo e demais actividades académicas.

Assumindo-se hoje o papel central do estudante no processo de ensino-aprendizagem, afirmação que nos parece com uma particular pertinência e aplicação no Ensino Superior em face da forma como se organizam o ensino e os demais espaços de formação-aprendizagem, importa analisar o grau de autonomia e de auto-regulação dos estudantes nas suas aprendizagens (Biggs, 1988; Entwistle, 1988; Rosário, 1999), por exemplo, os métodos de estudo implementados, a gestão do tempo e os comportamentos face à avaliação (Cone \& Owens, 1991; Pereira, 1997). A questão do rendimento académico passa também pelos conhecimentos prévios dos alunos (Hirsch, 1989; Marques \& Miranda, 1993; Rocco, 1981), nomeadamente quando o curso permite o acesso através de disciplinas específicas muito díspares ou aceita candidatos com classificações negativas. Logicamente que, sobretudo nestes casos, os professores desempenham um papel importante. A par da sua qualificação científica, interessa atender às suas competências pedagógicas e relacionais. Estas competências são importantes, quer na adaptação ao curso quer, e sobretudo, no sucesso académico dos estudantes, pois que se reflectem nos métodos de ensino e de avaliação dos professores (Grayson, 1993; Ramsden, 1988).

Descritas algumas variáveis associadas à aprendizagem e ao rendimento académico, o QVA ịnclui as percepções dos alunos em relação às seguintes dimensões: (i) bases de conhecimentos para o curso (inclui a preparação para as exigências do curso, as capacidades de leitura e escrita); (ii) métodos de estudo (inclui o acompanhamento das aulas, a consulta da bibliografia, a organização dos apontamentos); 
(iii) realização de exames (inclui comportamentos de tipo ansioso na preparação e realização dos testes, exames ou outras formas de avaliação); (iv) gestão do tempo (inclui itens sobre a planificação do tempo, o ritmo de prossecução das actividades, a realização dos trabalhos nos prazos fixados, a gestão equilibrada do tempo de estudo e de lazer); e, (v) relacionamento com os professores (inclui o diálogo com os professores, a possibilidade de contactos dentro e fora das aulas, a percepção da disponibilidade de tempo dos professores para os alunos, a apreciação dos seus processos de ensino-avaliação).

\section{Variáveis pessoais e de desenvolvimento psicossocial}

Para além das dimensões mais relacionadas com as questões da aprendizagem e do rendimento académico no Ensino Superior, tomados em sentido restrito, convém ainda salientar o papel e a importância de outro tipo de variáveis, mais directamente associadas ao self e ao desenvolvimento psicossocial dos estudantes. Aliás, e como vimos anteriormente, as tarefas com as quais os jovens são confrontados numa transição educativa não se circunscrevem exclusivamente às tarefas de índole curricular. Se quisermos, a par da adaptação às exigências académicas deste novo nível de ensino, o ingresso no Ensino Superior implica ainda, para um grande número de estudantes, o sair de casa, a separação dos amigos e das pessoas mais significativas, o confronto com um meio totalmente desconhecido, o decidir quem se é e o que se quer fazer da vida (Ferreira \& Hood, 1990). Questões como o estabelecimento de um forte sentido de identidade, o desenvolvimento de relações interpessoais mais maduras (com os pares, os professores e a família), a exploração de papéis sociais e sexuais, a resolução das questões da intimidade, assim como o estabelecimento de uma filosofia e plano de vida (incluindo a definição e o comprometimento com determinados objectivos pessoais e vocacionais), parecem assumir particular importância junto desta população.

Desta forma, e reconhecendo que as dimensões do domínio psicossocial desempenham um papel fundamental na compreensão e explicação da adaptação, do rendimento e do desenvolvimento experenciado pelos jovens ao longo da sua frequência do Ensino Superior, vários autores procuraram operacionalizar as tarefas desenvolvimentais com as quais os jovens são confrontados na sua transição para a universidade. Entre os autores que mais contribuíram para esta definição (Heath, 1968; Sanford, 1962; Chickering \& Reisser, 1993), Chickering (1969) foi um dos que assumiu maior destaque, apresentando-nos um modelo sobre o desenvolvimento psicossocial do estudante universitário, conceptualizado a partir de sete vectores: (i) tornar-se competente (desenvolver competências intelectuais, físicas e manuais, sociais e interpessoais); (ii) gerir as emoções (desenvolver competências para identificar e aceitar as emoções, assim como para expressá-las e controlá-las adequadamente); (iii) desenvolver a autonomia e a interdependência (reconhecimento e aceitação da importância da interdependência, desenvolvimento da independência 
emocional e da independência instrumental); (iv) desenvolver relações interpessoais maduras (estabelecimento de relacionamentos interpessoais que contribuam para o desenvolvimento de uma identidade, que inclui a aceitação e o aumento da tolerância às diferenças individuais, assim como a capacidade para estabelecer relações íntimas saudáveis); (v) estabelecer a identidade (estabelecimento de uma maior estabilidade e integração do self, aceitação pessoal, tanto no que se refere à imagem corporal como ao género e à orientação sexual, valorização pessoal, auto-estima e satisfação com os papéis de vida desempenhados); (vi) definir objectivos de vida (desenvolvimento de objectivos vocacionais claros e planos de acção para os implementar, realização de compromissos relativamente a interesses e actividades específicas e estabelecimento de compromissos interpessoais mais firmes); e (vii) desenvolver a integridade (aumento da congruência entre crenças e comportamentos e progressão de um sistema de valores rígido e moralista para um sistema mais humanizado e personalizado que reconhece e respeita os sistemas de valores dos outros). Para Chickering (1969) e Chickering \& Reisser (1993) cada um destes vectores acompanha o indivíduo ao longo de todo o seu ciclo de vida (podendo ter uma resolução positiva ou negativa) embora considerem que, em determinados períodos do desenvolvimento, alguns deles possam assumir particular destaque. Desta forma, consideram que as questões da competência, da autonomia e da identidade assumem particular importância durante os anos de frequência universitária.

Mas, se a experiência universitária conduz os estudantes a realizarem um movimento progressivo em direcção à tolerância, ao aumento da qualidade das relações interpessoais, ao desenvolvimento de um forte sentido de identidade e ao aumento da auto-confiança, obviamente que a qualidade da resolução destas tarefas depende, em muito, das próprias características pessoais que os alunos "trazem" para a universidade. Com efeito, tais características parecem estar na base, por exemplo, do melhor ou mais fraco aproveitamento das oportunidades que o próprio contexto universitário onde os estudantes estão inseridos lhes proporciona. Assim, alguns autores salientam dimensões psicológicas dos alunos, tais como estilos de coping (Billings \& Moos, 1981; Moos \& Schaefer, 1993; Polo, Hernández \& Pozo, 1996), a auto-estima (Burke, Midkiff \& Williams, 1985; Praeger \& Freeman, 1979), o locus de controlo (Crump, Hickson \& Laman, 1985; Prociuk \& Breen, 1977; Traub, 1982), os estilos atribucionais (Clifford, 1986; Platt, 1988; Powers, Douglas, Cool \& Gose, 1985), as percepções de auto-eficácia (Bento \& Ferreira, 1997; Lent, Brown \& Larkin, 1984; Multon, Brown \& Lent, 1991), a autonomia (Ferreira \& Castro, 1994), a congruência de interesses (Barak \& Rabbi, 1982; Henry \& Bardo, 1987), e a (in)decisão vocacional (Fuqua, Newman \& Seaworth, 1987; Gordan, 1995; Rose \& Elton, 1971; Soares, 1998; Taveira, 1997).

Procurando abarcar algumas destas variáveis, ditas mais associadas à pessoa e ao desenvolvimento psicossocial do estudante, o QVA contemplou na sua construção as seguintes dimensões: (i) autonomia (inclui a independência emocional de pais, colegas e outros, a gestão de projectos de vida, a tomada de iniciativa); (ii) autoconfiança (inclui as imagens e as expectativas pessoais em relação ao rendimento académico ou em relação à conclusão do curso, inferência das expectativas de colegas e professores a seu respeito); (iii) percepção pessoal de competência (inclui 
percepção de capacidades, as competências de resolução de problemas, a flexibilidade e profundidade de pensamento); (iv) desenvolvimento da carreira (inclui o investimento no curso, a elaboração de projectos, as perspectivas de realização profissional, a indecisão vocacional); (v) bem-estar psicológico (inclui a satisfação com a vida, o equilíbrio emocional, a estabilidade afectiva, a felicidade, o optimismo); e (vi) bem-estar físico (inclui aspectos relacionados com o sono e a alimentação, a saúde e o consumo de substâncias).

\section{Variáveis associadas ao contexto académico}

Finalmente, enunciaremos algumas variáveis mais associadas ao contexto académico enquanto novos vectores de análise da transição e adaptação académica dos estudantes. Tais variáveis, ditas contextuais do desenvolvimento e comportamento humanos, ajudam-nos a entender o papel do próprio campus na aprendizagem, adaptação e desenvolvimento psicossocial dos jovens universitários (Banning, 1989). Segundo alguns autores, os espaços físicos do campus (salas de aula, serviços, espaços de lazer, arquitectura e cores dos edifícios, arranjos e limpeza, etc.) afectam o comportamento dos alunos e o seu rendimento académico (McKee \& Witt, 1990; Gifford, 1997). Sugere-se, inclusive, que tais condições físicas, e mais concretamente o ratio de alunos por recursos disponíveis, condicionam a qualidade das relações e a socialização dos jovens (Hutt \& Varzey, 1966), as suas atitudes iniciais de envolvimento académico (Stern, 1986; Strange, 1996) ou os seus níveis de satisfação académica em geral (Weinstein, 1979).

As explicações para este impacto das variáveis do contexto são diversas. Segundo alguns autores as prestações e os comportamentos dos indivíduos exigem características físicas do setting adequadas (Barker, 1968). Por exemplo, a alta densidade de alunos na sala de aula pode fazer baixar o nível de interacção entre os alunos (Gifford, 1997), os seus níveis de atenção e de processamento da informação (Krantz \& Risley, 1972) e, ainda, aumentar as taxas de comportamentos agressivos e de tipo ansioso (Weinstein, 1979). Por sua vez, saindo dos espaços físicos de sala de aula, alguma investigação tem analisado as condições físicas das residências universitárias (Strange, 1996). Aqui, os sentimentos de espaços pessoais e de comunidade vivenciados pelos estudantes, associados a percepções de segurança, conforto e pertença, aparecem como variáveis igualmente importantes para descrever a adaptação e a realização académica dos estudantes (Anchors, Schroeder \& Jackson, 1978; Ender, Kane, Mable \& Strohm, 1980).

Todas estas variáveis do contexto acabam por facilitar ou comprometer os processos adaptativos dos alunos, ou outros recursos disponibilizados para esse efeito, desempenhando um papel decisivo na persistência dos alunos no curso e no seu envolvimento académico em geral (Baker \& Siryk, 1989; Hossler, 1984), ao mesmo tempo que são decisivos para o estabelecimento de novas relações e novos investimentos interpessoais por parte dos alunos (Grayson, 1993; Pereira, 1997; 
Winston et al., 1987). Se quisermos sair do campus, e da rede de espaços e serviços que lhe estão afectos, poderíamos mencionar ainda outras variáveis do contexto, como a rede de transportes, a dimensão da cidade e a própria estrutura familiar do estudante. Por exemplo, vários estudos mencionam a importância destas relações para o ajustamento académico dos estudantes, mesmo quando falamos de jovens adultos (Armsden \& Geenberg, 1989; Albert, 1988; Lopez et al., 1988; Wick \& Shilkret, 1986).

Várias subescalas do QVA procuram atender a algumas destas variáveis de contexto enunciadas: (i) adaptação à instituição (inclui o sentir-se bem ou mal na instituição, adaptação aos horários e ao funcionamento dos serviços, gosto pela instituição que frequenta e pelo ambiente circundante); (ii) adaptação ao curso (inclui o gosto e a satisfação pelo curso, a percepção da sua organização e da qualidade das suas disciplinas, a ligação possível entre os conteúdos curriculares e as saídas profissionais); (iii) envolvimento em actividades extracurriculares (inclui a participação em iniciativas associativas, em actividades culturais e recreativas, a pertença em órgãos associativos); (iv) relacionamento com os colegas (inclui as amizades, a expressão de sentimentos, a tolerância intercultural, a cooperação com os pares); (v) relacionamento com a família (inclui o relacionamento com os pais, o apoio recebido, a necessidade de idas a casa, o diálogo familiar em torno dos projectos pessoais e das escolhas vocacionais); $\mathrm{e}$ (vi) gestão dos recursos económicos (inclui referências à situação económica do aluno, problemas na gestão das verbas auferidas, situações de compromisso entre verbas disponíveis e envolvimento em actividades extracurriculares, necessidade de trabalho em part-time).

\section{Metodologia}

\section{Amostra}

A amostra deste estudo foi constituída por 1273 estudantes a frequentar o primeiro ano de 41 licenciaturas da Universidade do Minho(UM). A maioria dos elementos da amostra é do sexo feminino (61,5\% raparigas e 38,5\% rapazes) e as suas idades oscilam entre os 17 ( 5 alunos) e os 52 anos de idade ( 1 aluno), aproximando-se a média, em ambos os sexos, dos 20 anos. A grande maioria dos sujeitos da amostra são solteiros $(95,2 \%)$ e residem em Braga ou nas suas redondezas no período de aulas (93,8\%), embora cerca $50 \%$ não vivam com a sua família. A quase totalidade dos alunos da amostra encontra-se apenas a estudar $(87,1 \%)$, havendo $7,7 \%$ e $4 \%$ com um part-time ou um emprego a tempo inteiro, respectivamente. O curso/estabelecimento de ensino que frequentam corresponde, para a maioria dos elementos da amostra (59\%), à sua $1 .^{a}$ opção no processo de candidatura ao Ensino Superior. Mesmo assim, cerca de um quarto dos alunos da amostra não se encontra a frequentar um curso que correspondesse à sua $1 .^{\mathrm{a}}$ ou 2. ${ }^{\mathrm{a}}$ opção. Aliás, no início do 2 . $^{\circ}$ semestre e aquando da administração do questionário, $74,7 \%$ dos alunos 
mostram-se satisfeitos com o curso que frequentam e 84,8\% têm também essa opinião favorável em relação à universidade.

\section{Instrumento}

O Questionário de Vivências Académicas (QVA) é constituído por 170 itens, distribuídos por 17 subescalas, como se pode constatar no quadro 1 , que cobrem dimensões pessoais, relacionais e institucionais da adaptação dos estudantes ao contexto universitário (Almeida \& Ferreira, 1997). Para cada um dos itens que compõem o instrumento os sujeitos deverão decidir o seu grau de acordo/desacordo para cada uma das situações apresentadas, numa escala de tipo likert de cinco pontos. Acrescente-se que se trata de um questionário orientado para o despiste (screening) de eventuais dificuldades vivenciadas pelos estudantes na sua inserção e adaptação académica, ou seja, não estamos face a um instrumento de diagnóstico, muito menos de diagnóstico psicológico.

\section{Procedimento}

O Questionário de Vivências Académicas (QVA) foi administrado colectivamente, em ambiente de sala de aula, na parte final de um tempo lectivo cedido pelos professores dos cursos envolvidos na amostra. Para cada curso, procurou-se ir às disciplinas com maior frequência e às respectivas aulas teóricas. Após a explicação dos objectivos do questionário, e feito o pedido de colaboração aos estudantes, deixava-se-lhes total liberdade de aceitar ou não participar. Explicava-se também que o questionário seria anónimo e os seus dados confidenciais, sendo apenas necessário preencher alguns elementos de identificação para caracterização da amostra (cerca de $20 \%$ dos estudantes não aceitaram colaborar, apontando razões diversas como, por exemplo, a necessidade de apanharem um transporte ou o facto de não serem alunos do primeiro ano). De um modo geral, os alunos que responderam ao QVA fizeram-no de forma bastante consciente e interessada. A aplicação não teve limite de tempo e os alunos demoraram, em média, 20 minutos a responder ao questionário.

\section{Resultados}

Apresentam-se no quadro 2 os resultados nas 17 subescalas do questionário. Esta apresentação considera, a par da média e do desvio-padrão, alguns índices da distribuição dos resultados (variação, assimetria e curtose). Importa referir que, face à organização das respostas, as pontuações mais elevadas nas subescalas correspondem a melhores percepções dos alunos. Esta lógica, no entanto, merece ser 
Quadro 1 Dimensōes do Questionário de Vivências Académicas (QVA)

\begin{tabular}{|c|c|}
\hline Dimensões & Definição \\
\hline $\begin{array}{l}\text { Bases de conhecimentos para o } \\
\text { curso }\end{array}$ & $\begin{array}{l}\text { Inclui as bases de conhecimentos, a preparação para as exigências do } \\
\text { curso, as capacidades de leitura e escrita de textos. }\end{array}$ \\
\hline Métodos de estudo & $\begin{array}{l}\text { Inclui o acompanhamento das aulas, a consulta da bibliografia, a } \\
\text { auto-regulação na aprendizagem, a organização dos apontamentos. }\end{array}$ \\
\hline Realização de exames & $\begin{array}{l}\text { Inclui comportamentos de preparação e realização dos testes, exames ou } \\
\text { outras formas de avaliação. Tais comportamentos são avaliados, nesta } \\
\text { escala, por vivências de tipo ansioso. }\end{array}$ \\
\hline Gestão do tempo & $\begin{array}{l}\text { Inclui itens sobre a planificação do tempo, o ritmo de prossecução das } \\
\text { actividades, a realização dos trabalhos nos prazos fixados, a gestão do } \\
\text { tempo de estudo e de lazer. }\end{array}$ \\
\hline Relacionamento com os professores & $\begin{array}{l}\text { Inclui o diálogo com os professores, a possibilidade de contactos dentro e } \\
\text { fora das aulas, a percepção da disponibilidade de tempo dos professores } \\
\text { para os alunos. }\end{array}$ \\
\hline Autonomia & $\begin{array}{l}\text { Inclui a independência emocional de pais, colegas e outros, a gestăo de } \\
\text { projectos de vida, a tomada de iniciativa. }\end{array}$ \\
\hline Autoconfiança & $\begin{array}{l}\text { Inclui as imagens e as expectativas pessoais em relação ao rendimento } \\
\text { académico ou em relaçăo à conclusão do curso, inferência das } \\
\text { expectativas de colegas e professores a seu respeito. }\end{array}$ \\
\hline Percepção pessoal de competência & $\begin{array}{l}\text { Inclui a percepção de capacidades, as competências de resolução de } \\
\text { problemas, a flexibilidade e profundidade de pensamento. }\end{array}$ \\
\hline Desenvolvimento da carreira & $\begin{array}{l}\text { Inclui o investimento no curso, a elaboração de projectos, as perspectivas } \\
\text { de realização profissional, a decisão vocacional. }\end{array}$ \\
\hline Bem-estar psicológico & $\begin{array}{l}\text { Inclui a satisfação com a vida, o equilíbrio emocional, a estabilidade } \\
\text { afectiva, a felicidade, o optimismo. }\end{array}$ \\
\hline Bem-estar físico & $\begin{array}{l}\text { Inclui aspectos relacionados com o sono e a alimentação, a saúde, o } \\
\text { consumo de substâncias. }\end{array}$ \\
\hline Adaptação à instituição & $\begin{array}{l}\text { Inclui o sentir-se bem ou mal na instituição, adaptação aos horários e ao } \\
\text { funcionamento dos serviços, gosto pela instituição que frequenta e pelo } \\
\text { ambiente circundante. }\end{array}$ \\
\hline Adaptação ao curso & $\begin{array}{l}\text { Inclui o gosto e a satisfação pelo curso, a percepção da sua organizaçăo } \\
\text { e da qualidade das suas disciplinas, a ligação possível entre conteúdos } \\
\text { curriculares e saídas profissionais. }\end{array}$ \\
\hline $\begin{array}{l}\text { Envolvimento em actividades } \\
\text { extracurriculares }\end{array}$ & $\begin{array}{l}\text { Inclui a participação em iniciativas associativas, em actividades culturais e } \\
\text { recreativas, a prática desportiva. }\end{array}$ \\
\hline Relacionamento com os colegas & $\begin{array}{l}\text { Inclui as amizades, a expressão de sentimentos, a tolerância intercultural, } \\
\text { a cooperação com os pares. }\end{array}$ \\
\hline Relacionamento com a família & $\begin{array}{l}\text { Inclui o relacionamento com os pais, o apoio recebido, a necessidade de } \\
\text { idas a casa, o diálogo em torno dos projectos pessoais e das escolhas } \\
\text { vocacionais. }\end{array}$ \\
\hline Gestão dos recursos económicos & $\begin{array}{l}\text { Inclui referências à situação económica do aluno, problemas na gestäo } \\
\text { das verbas auferidas, situaçōes de compromisso entre verbas disponíveis } \\
\text { e envolvimento em actividades extracurriculares. }\end{array}$ \\
\hline
\end{tabular}


Quadro 2 Estatistica descritiva dos resultados por dimensoes do QVA

\begin{tabular}{|c|c|c|c|c|c|c|c|c|}
\hline Dimensto & $N^{*}$ itens & Média & $\begin{array}{c}\text { Desvio- } \\
\text { Padr. }\end{array}$ & Min. & Max. & Assim. & Curt. & Alfa \\
\hline Bases de conhecimentos para o curso & 6 & 20,1 & 3,90 & 7 & 30 & 0,01 & 0,00 & 0.78 \\
\hline Métodos de estudo & 11 & 36,3 & 5,51 & 18 & 53 & $-0,01$ & 0,19 & 0,76 \\
\hline Realizaclo de exames & 10 & 30,8 & 5.92 & 10 & 49 & $-0,14$ & 0.39 & 0.78 \\
\hline Gestalo do tempo & 8 & 26,1 & 4,90 & 9 & 39 & $-0,13$ & 0,41 & 0.72 \\
\hline Relacionamento com os prolessores & 14 & 40,5 & 7,20 & 17 & 67 & 0,07 & 0.21 & 0.79 \\
\hline Autonomia & 12 & 42,3 & 6,35 & 19 & 60 & $-0,11$ & 0,01 & 0,76 \\
\hline Autoconfiança & 12 & 40,8 & 6,44 & 16 & 58 & $-0,30$ & 0,40 & 0,80 \\
\hline Percepçăo pessoal de competência & 10 & 34,3 & 5,07 & 14 & 49 & $-0,04$ & 0,43 & 0,75 \\
\hline Desenvolvimento da carreira & 14 & 49,0 & 8,50 & 20 & 70 & $-0,18$ & -0.09 & 0,85 \\
\hline Bem-estar psicológico & 14 & 45,8 & 9,55 & 18 & 68 & $-0,18$ & $-0,14$ & 0,88 \\
\hline Bem-estar físico & 13 & 50,0 & 7,19 & 22 & 65 & -0.56 & 0,39 & 0.79 \\
\hline Adaptaça à instituię̧ & 11 & 42,5 & 6,11 & 18 & 55 & $-0,55$ & 0,40 & 0,74 \\
\hline Adaptaçăo ao curso & 15 & 51,9 & 9,08 & 20 & 74 & $-0,29$ & $-0,01$ & 0,85 \\
\hline Envolvimento act. extracurriculares & 11 & 33,3 & 5.93 & 15 & 53 & 0.27 & 0,01 & 0.69 \\
\hline Rolacionamento com os colegas & 15 & 56,8 & 8,71 & 22 & 75 & $-0,32$ & $-0,03$ & 0.87 \\
\hline Relacionamento com a familia & 10 & 39,9 & 6,32 & 15 & 50 & $-0,82$ & 0,66 & 0,82 \\
\hline Gestao dos recursos económicos & 8 & 27,1 & 6.06 & 8 & 40 & $-0,23$ & $-0,18$ & 0.83 \\
\hline
\end{tabular}

discutida em relação a duas subescalas: relacionamento com a familia e gestão dos recursos económicos. Na subescala relacionamento com a familia, as notas mais elevadas traduzem maior interdependência entre o jovem e a sua familia (diríamos uma situação de compromisso entre a dependência e a independência familiar absolutas). Neste caso, o jovem torna-se autónomo, mas mantém relaçōes de proximidade afectiva e social com os familiares. Na subescala gestão dos recursos económicos já não é tăo claro o sentido a dar às pontuações. As pontuaçōes nalguns itens reportam-se essencialmente a dificuldades na gestāo das verbas auferidas, enquanto noutros se avaliam situações económicas difíceis vividas pelo estudante.

A leitura das médias e dos desvios-padrão obtidos não é fácil no presente, uma vez que nos faltam parâmetros de referência em termos nacionais. Mesmo assim, atendendo à escala de resposta aos itens (likert de cinco pontos), verificamos que, na generalidade das subescalas, a média de resposta dos alunos se situou acima, ou ligeiramente acima, de uma pontuação intermédia, considerando o número de itens por cada subescala. Os valores mínimos e máximos da distribuição nas 17 subescalas distanciam-se entre si em mais de quatro unidades de desvio-padrão, o que nos elucida sobre as boas capacidades discriminativas dos itens incluídos. Finalmente, os coeficientes de consistência interna dos itens (alfa de Cronbach) apresentam valores aceitáveis, e apenas a subescala envolvimento em actividades extracurriculares apresenta um coeficiente inferior a 0,70 (situou-se em 0,69).

A dimensionalidade do QVA foi analisada através de uma análise factorial exploratória. Optou-se por uma análise em componentes principais com rotação varimax, tomando todos os factores com valor-próprio (eigen-value) igual ou superior à unidade. No quadro 3 apresentam-se as saturaçס̄es factoriais das 17 subescalas do QVA (apenas as saturações de valor igual ou superior a 0,40 ).

Os resultados do estudo da dimensionalidade do QVA evidenciam uma 
Quadro 3 Análise factorial das 17 subescalas do QVA

\begin{tabular}{|c|c|c|c|c|c|}
\hline Dimensão & Factor I & Factor II & Factor III & Factor IV & Factor $\mathrm{V}$ \\
\hline Bases de conhecimentos para o curso & 0,45 & 0,68 & - & - & - \\
\hline Métodos de estudo & - & - & - & 0,73 & - \\
\hline Realização de exames & 0,86 & - & - & - & - \\
\hline Gestão do tempo & - & - & - & 0,80 & - \\
\hline Relacionamento com os professores & - & - & - & 0,46 & - \\
\hline Autonomia & 0,61 & - & 0,40 & - & - \\
\hline Autoconfiança & 0,80 & - & - & - & - \\
\hline Percepção pessoal de competência & 0,61 & 0,58 & - & - & - \\
\hline Desenvolvimento da carreira & - & 0,83 & - & - & - \\
\hline Bem-estar psicológico & 0,76 & - & - & - & - \\
\hline Bem-estar físico & 0,62 & - & - & - & - \\
\hline Adaptação à instituição & - & - & 0,77 & - & - \\
\hline Adaptação ao curso & - & 0,77 & - & - & - \\
\hline Envolvimento act. extracurriculares & - & - & 0,81 & - & - \\
\hline Relacionamento com os colegas & - & - & 0,78 & - & - \\
\hline Relacionamento com a família & - & - & - & 0,42 & 0,57 \\
\hline Gestão dos recursos económicos & 一 & 一 & 一 & - & 0,77 \\
\hline Valor-próprio & 70,04 & 10,56 & 10,48 & 10,23 & 10,00 \\
\hline Percentagem da variância & 41,04 & 9,10 & 8,07 & 6,06 & 5,09 \\
\hline
\end{tabular}

estrutura assente em cinco factores que explicam $72 \%$ da variância dos resultados nas 17 subescalas.

Como se pode constatar no quadro 3, o factor I assume bastante importância ( $41 \%$ da variância), encontrando-se sobretudo associado às dimensões de natureza mais pessoal. Algumas destas dimensões cobrem áreas do desenvolvimento psicológico (por exemplo, autonomia, autoconfiança e bem-estar físico e psicológico), enquanto outras se reportam à realização académica (percepção de capacidades, bases de conhecimentos e ansiedade na realização de testes). O factor II (responsável por $9,1 \%$ dos resultados) está mais directamente relacionado com as dimensões do projecto vocacional do estudante (carreira), onde a adaptação ao curso entra obviamente. $\mathrm{O}$ factor III reúne as dimensões mais directamente associadas à adaptação do aluno ao contexto académico, entendido aqui em sentido lato (adaptação à universidade, relacionamento com os colegas e envolvimento em actividades extracurriculares). O factor IV, ainda associado à realização académica, reúne as dimensões do QVA mais directamente relacionadas com o processo de ensino-aprendizagem, ou seja, as capacidades do estudante em organizar o estudo e em gerir o tempo, assim como o relacionamento com os professores. A importância destes aspectos, e a sua saturação num novo factor de índole académica, poderá induzir-nos para a singularidade destas variáveis, bastante confinadas às práticas de estudo e à aprendizagem dos estudantes, ao que não será alheio o facto de estarmos a lidar com alunos do primeiro ano vivenciando mudanças nos processos de ensino-aprendizagem. Finalmente, o factor $\mathrm{V}$ parece reflectir uma vertente económico-familiar de suporte ao aluno, tratando-se já de um factor bastante específico. 


\section{Discussão e conclusões}

Um conjunto bastante diverso de variáveis interfer na qualidade da transição e adaptação dos estudantes ao Ensino Superior. A literatura agrupa as variáveis seguindo diferentes taxonomias. Neste artigo descrevemos esse processo de transição e adaptação recorrendo a: (i) variáveis mais directamente relacionadas com as tarefas de aprendizagem e de realização académica; (ii) variáveis de índole pessoal mais relacionadas com o desenvolvimento psicossocial do estudante; e (iii) variáveis mais amplas, associadas ao contexto académico. Todas estas variáveis interactuam, para explicar a qualidade da transição/adaptação, reconhecendo-se na literatura que toda a transição é vivenciada e faz apelo quer aos recursos psicológicos do próprio sujeito, quer às condições/exigências colocadas pelo novo contexto onde o indivíduo se vai inserir.

Tendo em vista uma avaliação inicial (despiste) de um conjunto de variáveis associadas às vivências que os alunos podem experienciar enquanto recém-chegados ao Ensino Superior ("caloiros"), procedeu-se à construção do Questionário de Vivências Académicas (QVA). As 17 subescalas que compõem o instrumento mostram uma boa dispersão dos resultados, conseguindo-se também índices satisfatórios de consistência interna dos respectivos itens. Os coeficientes alfa oscilaram entre 0,69 (envolvimento em actividades extracurriculares) e 0,88 (bem-estar psicológico). Se neste último caso podemos associar o índice obtido ao maior número de itens da subescala $(n=14)$, em relação ao envolvimento em actividades extracurriculares, a diversidade de situações que compõem esta subescala e o facto de os alunos do primeiro ano poderem desconhecer, ainda, algumas das alternativas de ocupação dos tempos livres proporcionadas pelo campus, poderão explicar a menor homogeneidade verificada nas respostas aos itens desta subescala.

A análise factorial aponta para a existência de cinco factores que explicam, no seu conjunto, $72 \%$ da variância dos resultados nas 17 subescalas. O primeiro factor reúne as dimensões pessoais e mais directamente ligadas ao desenvolvimento psicossocial do estudante, assim como a ansiedade na avaliação (realização de exames) tida, na versão inicial do QVA, como uma subescala da área da aprendizagem e do rendimento académico. É possível que os níveis de ansiedade experenciados pelos sujeitos nas situações de avaliação estejam mais associados às suas características pessoais ou de personalidade do que aos próprios contextos de aprendizagem e realização. $\mathrm{O}$ factor II prende-se com as subescalas académicas relacionadas mais com a adaptação ao curso e desenvolvimento da carreira socioprofissional, do que com as questões mais directamente relacionadas com o ensino, o estudo e a aprendizagem (factor IV). A delimitação na análise factorial destes dois factores na área académica não estava prevista inicialmente na fundamentação do QVA, mas acaba por se entender se considerarmos as dificuldades particulares que os alunos do primeiro ano podem vivenciar na sua adaptação a novas formas de ensinar e avaliar, a novos padrões de relacionamento com os professores, a mais tempo para gerir ou a novos métodos de estudo a construir.

$\mathrm{O}$ factor III congrega as subescalas mais directamente relacionadas com o 
contexto académico, pontuando aqui a adaptação à instituição, o envolvimento em actividades extracurriculares e o relacionamento com colegas. Finalmente, o factor V integra apenas duas subescalas: gestão de recursos económicos e relacionamento com a família. Trata-se de um factor não previsto inicialmente e reunindo duas subescalas que, à partida, não eram supostas andarem juntas. Lendo os dados à posteriori parece-nos que este factor poderá traduzir as necessidades de subsistência e/ou de apoio dos estudantes, sobretudo nos primeiros meses em que vivem afastados da família, gerindo verbas mensais e equilibrando despesas de subsistência com despesas de lazer.

A finalizar, os coeficientes estatísticos obtidos legitimam a utilização do questionário servindo um primeiro nível de avaliação de variáveis que, de acordo com a literatura, parecem explicar os níveis e a qualidade da transição e adaptação ao Ensino Superior. Evidentemente que tais índices, mesmo sendo adequados, não legitimam a suficiência do QVA para uma avaliação, dita diagnóstica, das várias dimensões psicossociais versadas. É pois importante respeitar o QVA enquanto um instrumento de despiste, provavelmente com maior interesse de utilização para a investigação do que para a intervenção.

\section{Notas}

1 O Ensino Superior em Portugal subdivide-se em: Ensino Universitário e Ensino Politécnico. Neste trabalho não os diferenciamos, muito embora a maioria dos estudos nesta área se reportem genericamente à universidade.

2 As transições caracterizam-se por serem períodos de conflitos de papéis entre dois estádios da vida claramente definidos e por implicarem o estabelecimento de novos padrões de vida. Desta forma, os acontecimentos de vida (life events) só são considerados transições se, face a eles, os indivíduos experenciarem um sentido de descontinuidade pessoal e uma necessidade de desenvolverem novos padrões de resposta comportamental, cognitiva e afectiva. Transição é, neste sentido, não o acontecimento em si, mas a percepção individual de mudança. No presente trabalho, assumimos o ingresso no Ensino Superior como transição, dado considerarmos que este acontecimento de vida encerra, em si mesmo, fortes possibilidades de ser experienciado enquanto transição, dado comportar, a par de um conjunto de mudanças desenvolvimentais, toda uma série de novos e complexos desafios que "forçam" o estudante universitário a um questionamento pessoal nos vários domínios da sua existência (académico, vocacional, social, emocional, intelectual e ético), promovendo assim o estabelecimento de novos padrões de funcionamento. 


\section{Referências}

Albert, M. M. (1988). Physical and psychological separation of late adolescents from their parents as it relates to adjustment to college. Dissertação de doutoramento. Miami Shores, FL: Barry University.

Almeida, S. L. \& Ferreira, J. A. (1997). Questionário de Vivências Académicas. Braga: Universidade do Minho.

Almeida, L. S., Soares, A. P. C. \& Ferreira, J. A. G. (1999). Adaptação, rendimento e desenvolvimento dos estudantes no ensino superior: Construção/validação do Questionário de Vivências Académicas. Relatórios de Investigação. Braga: Universidade do Minho, Centro de Estudos em Educação e Psicologia.

Anchors, S., Schroeder, C. \& Jackson, S. (1978). Making yourself at home: A practical guide to restructuring and personalizing your residence hall environment. Washington, DC: ACPA Media publications.

Armsden, G. \& Geenberg, M. (1989). Inventory of Parent and Peer Attachment: Preliminary Test Manual. Seattle, WA: University of Washington.

Arroteia, J. C. (1996). O ensino superior em Portugal. Aveiro: Universidade de Aveiro.

Astin, A. (1975). Preventing students from dropping out. São Francisco: Jossey-Bass Publishers.

Astin, A. (1984). Student involvement: A developmental theory for higher education. Journal of College Student Personnel, 25, 297-308.

Astin, A. (1993). What matters in college? Four critical years revised. São Francisco: Jossey-Bass Publishers.

Baker, R. W. \& Siryk, B. (1989). Student Adaptation to College Questionnaire (SACQ): Manual. Los Angeles, CA: Western Psychological Services.

Baker, R., McNeil, O. \& Siryk, B. (1985). Expectations and reality in freshman adjustment to college. Journal of Counseling Psychology, 32, 94-103.

Banning, J. H. (1989). Impact of college environments on freshman students. In M. L. Upcraft \& J. Gardner (Eds.), The freshman year experience: Helping students survive and succeed in college. São Francisco: Jossey-Bass Plublishers.

Barak, A. \& Rabbi (1982). Predicting persistence, stability and achivement in college by major choice consistency: A test of Holland's hypothesis. Journal of Vocational Behaviour, 20, 235-243.

Barker, R. G. (1968). Ecological psychology. Stanford, CA: Stanford University Press.

Bastos, A. (1998). Desenvolvimento pessoal e mudança em estudantes do ensino superior: Contributos da teoria, investigação e prática, Dissertação de doutoramento. Braga: Universidade do Minho.

Bastos, A. \& Gonçalves, O. F. (1996). Intervenção psicológica no ensino superior: Construção, implementação e avaliação de um programa de desenvolvimento pessoal. Intervenção Psicológica: Teoria, Investigação e Prática, 1, 195-206.

Belo, S. I. (1999). Autoconceito e adaptação à universidade em alunos do $1 .^{\circ}$ ano, Dissertação de mestrado. Braga: Universidade do Minho.

Bento, F. \& Ferreira, J. A. (1997). Autoconceito e participação social do estudante do ensino superior. In Actas da Conferência Internacional "A informação e a orientação 
escolar e profissional no ensino superior: Um desafio da Europa". Coimbra: Universidade de Coimbra.

Biggs, J. B. (1988). Assessing study approaches to learning. Australian Psychologist, 23, 197-206.

Billings, A. G. \& Moos, R. H. (1981). The role of coping responses and social resources in attenuatting the stress of life events. Journal of Behavioural Medicine, 4, 139-157.

Braga da Cruz, M., Cruzeiro, M. E., Ramos, A., Leandro, E., Nunes, J. S., Matias, N., Pedroso, P., Robinson, M. G. \& Cavaco, V. (1995). O desenvolvimento do ensino superior em Portugal: Situação e problemas de acesso. Lisboa: Departamento de Programação e Gestão Financeira, Ministério da Educação.

Burke, J. P., Midkiff, R. M. \& Williams, R. V. (1985). The role of self-esteem in affective reactions to achievement-related situations. Educational and Psychological Research, 5, 191-203.

Carneiro, J. P. (1999). Adaptação à universidade e rendimento académico em alunos do $1 .^{\circ}$ ano, Dissertação de mestrado. Braga: Universidade do Minho.

Chickering, A. W. (1969). Education and identity. San Francisco: Jossey-Bass.

Chickering, A. W. \& Reisser (1993). Education and identity (2. ${ }^{a}$ ed.). São Francisco: Jossey-Bass Publishers.

Conceição, P., Durão, D. F. G., Heitor, M. V. \& Santos, F. (1998). Novas ideias para a Universidade. Lisboa: Instituto Superior Técnico.

Cone, A. \& Owens, S. K. (1991). Academic and locus of control enhancement in a freshman study skills and college adjustment course. Psychological Reports, 68, 1211-1217.

Costa, M. E. (1991). Contextos sociais de vida e desenvolvimento da identidade. Porto: INIC.

Costa, M. E. \& Campos, B. P. (1986). Identidade de estudantes universitários: Diferenças de curso e sexo. Cadernos de Consulta Psicológica, 2, 5-11.

Clifford, M. M. (1986). The comparative effects of strategy and effort attributions. British Journal of Educational Psychology, 56, 75-83.

Creamer, D. G. (1990). College student development: Theory and practice for the 1990's. Amer College Personnel.

Crump, B. R., Hickson, J. H. \& Laman, A. (1985). Relationship of locus of control to achievement and self-concept in education majors. Psychological Reports, 57, 1055-1060.

Diniz, A. M. \& Almeida, L. (1997). Construção de uma escala de qualidade da integração no ensino superior (EQIES). Psicologia: Teoria, Investigação e Prática, 2, 85-96.

Education Training Youth (1995). Key data on education in the European Union. Comissão Europeia.

Ender, K., Kane, N., Mable, P. \& Strohm, M. (1980). Creating community in residence halls. Washington, DC: ACPA Media publications.

Entwistle, N. J. (1988). La comprensión del aprendizaje en el aula. Barcelona: Paidós/M. E. C.

Evans, N. J., Forney, D. S. \& Dibrito, F. G. (1998). Student development in college: Theory, research and practice. São Francisco: Jossey-Bass Publishers.

Faria, L. \& Santos, N. L. (1998). Validação de uma escala de concepções pessoais de competência no contexto universitário. Actas do IV Congresso Galaico-Português de Psicopedagogia. Braga: Universidade do Minho. 
Ferreira, J. A. \& Castro, M. T. (1994). A adaptação do Inventário de Desenvolvimento da Autonomia de Iowa com jovens universitários. Psychologica, 12, 143-153.

Ferreira, J. A. \& Hood, A. (1990). Para a compreensão do desenvolvimento psicossocial do estudante universitário. Revista Portuguesa de Pedagogia, XXIV, 391-406.

Fuqua, D. R., Newman, J. L. \& Seaworth T. B. (1987). The relationship of career indecision and anxiety: A multivariate examination. Journal of Vocational Behaviour, 30, 175-186.

Gago, J. M., Amaral, J. F., Grácio, S., Rodrigues, M. J., Fernandes, L., Ruivo, B., Ambrósio, T., Silva, C. M., Duarte, T., Teixeira, M., Proença, L., Alves, M. G. \& Lisboa, M. (1994). Prospectiva do ensino superior em Portugal. Lisboa: Departamento de Programação e Gestão Financeira, Ministério da Educação.

Gifford, R. (1997). Environmental psychology: Principles and practice. Needham Heights: Allyn \& Bacon.

Gonçalves, O. F. \& Cruz, J. F. (1988). A organização e implementação de serviços universitários de consulta psicológica e desenvolvimento humano. Revista Portuguesa de Educação, 1(1), 127-145.

Gordan, V. N. (1995). The undecided college student: An academic and career advising challenge. (2. ${ }^{\mathrm{a}}$ ed.). Springfield: Charles Thomas Publisher.

Grayson, A. (1993). Accounting for help-seeking: Why are some students reluctant to approach lecturers for help?. Dissertação de doutoramento. Nottingham Trent University.

Heath, D. (1968). Growing up in college. São Francisco: Jossey-Bass Publishers.

Henry, P. \& Bardo, H. R. (1987). Expressed occupational choice of nontraditional premedical students as measured by the Self-Directed Search: An investigation of Holland's theory. Psychological Reports, 60, 575-581.

Herr, E. L. \& Cramer, S. H. (1992). Career guidance and counseling through the life span. Systematic approaches. Nova Iorque: Harper Gollins Publishers.

Hirsch, E. D. (1989). The primal scene of education. New York Review of Books, 36, 29-34.

Hood, A. \& Ferreira, J. A. (1983). Stages in the cognitive development of university students. Revista Portuguesa de Pedagogia, XVII, 79-90.

Hossler, D. (1984). Enrollment management. Nova Iorque: College Entrance Examination Board.

Hutt, C. \& Varzey, M. J. (1966). Differential effects of group density on social behavior. Nature, 209, 1371-1372.

Komives, S. R., Delworth, U. \& Woodard, D. (1996). Student services: A handbook for the profession. São Francisco: Jossey-Bass Publishers.

Krantz, P. \& Risley, T. (1972). The organization of group care environments: Behavioural ecology in the classroom. Lawrence: Kansas University.

Leitão, L. M. \& Paixão, M. P. (1999). Contributos para um modelo integrado de orientação escolar e profissional no ensino superior. Psicologia: Teoria, Investigação e Prática, 1, 191-209.

Lent, R. W., Brown, S. D. \& Larkin, K. C. (1984). Relation of self-efficacy expectations to academic achievement and persistence. Journal of Counseling Psychology, 31, 356-362.

Lopez, F., Campbell, V. \& Watkins, C. E. (1988). Family structure, psychological separation and college adjustment: A canonical analysis and cross-validation. Journal of Counseling Psychology, 35, 402-409. 
Lowenthal, M. F. \& Pierce, R. (1975). The pretransitional stance. In M. F. Lowenthal, M. Thurnher \& D. Chiriboga (Eds.), Four stages of life: A comparative study of men and women facing transitions. São Francisco: Jossey Bass Piblishers.

Marques, J. F. \& Miranda, M. J. (1993). Sobre o acesso ao ensino superior em Portugal: Estudo de indicadores numa amostra de estudantes da Universidade de Lisboa. Revista Portuguesa de Psicologia, 29, 111-140.

Martins, F. (1998). A satisfação académica: Construção de uma escala. Actas do IV Congresso Galaico-Português de Psicopedagogia. Braga: Universidade do Minho.

McKee, W. T. \& Witt, J. C. (1990). Effective teaching: A review of instructional and environmental variables. In T. B. Gutkin \& C. R. Reynolds (Eds.), The handbook of school psychology. Nova Iorque: Wiley.

Medeiros, T. A., Ferreira, J. A. \& Ponciano, E. (1997). Caracterização do estudante da Universidade dos Açores. Comunicação apresentada no I Encontro de Serviços de Apoio Psicológico no Ensino Superior. Lisboa: Instituto Superior Técnico.

Menezes, I., Costa, M. E. \& Campos, B. P. (1989). Valores de estudantes universitários. Cadernos de Consulta Psicológica, 5, 53-68.

Miranda, M. J. (1994). Estudo do Inventário de Estilos de Pensamento com estudantes universitários: Dados metrológicos. Psychologica, 12, 131-141.

Moos, R. H. \& Schaefer, J. A. (1993). Coping resources and processes: Currents concepts and measures. In L. Goldberg \& S. Breznitz (Eds.), Handbook of stress: Theoretical and clinical aspects (2. ${ }^{\mathrm{a}}$ ed., pp. 234-257). Nova Iorque: Free Press.

Multon, K. D., Brown, S. D. \& Lent, R. W. (1991). Relation of self-efficacy beliefs to academic outcomes: A meta-analytic investigation. Journal of Counseling Psychology, $38,30-39$

Neugarten, B. L., Moore, J. C. \& Lowe, J. C. (1965). Age nouns, age constrains and adult socialization. American Journal of Sociology, 70, 710-717.

Nico, J. B. (1995). A relação pedagógica na universidade: Ser-se caloiro, Dissertação de mestrado. Lisboa: FPCEUL.

Pace, C. (1984). Measuring the quality of college student experiences. Los Angeles: University of California, Higher Education Research Institute.

Pascarella, E. T. \& Terenzini, P. T. (1991). How college affects students: Findings and insights from twenty years of research. Sao Francisco: Jossey-Bass Publishers.

Pearlin, L. I. (1982). Discontinuities in the study of aging. In T. K. Haraven \& K. J. Adams (Eds.), Aging and life course transitions: An interdisciplinary perspective. Nova Iorque: Guilford Press.

Pereira, A. M. S. (1997). Helping student cope: Peer counseling in higher education, Dissertação de doutoramento. Hull: University of Hull.

Platt, C. W. (1988). Effects of causal attributions for success on first-term college performance: A covariance structure model. Journal of Educational Psychology, 80, 569-578.

Polo, A., Hernández, J. M. \& Pozo, C. (1996). Evaluación del estrés académico en estudiantes universitários. Ansiedad y Estrés, 2(2-3), 159-172.

Powers, S., Douglas, P., Cool, B. A. \& Gose, K. F. (1985). Achievement motivation and attributions for success and failure. Psychological Reports, 57, 751-754.

Praeger, K. J. \& Freeman, A. (1979). Self-esteem, academic competence, educational 
aspiration and curriculum choice of urban community college students. Journal of College Student Personnel, 5, 392-397.

Prociuk, T. J. \& Breen, L. J. (1977). Internal-external locus of control and information seeking in a college academic situation. Journal of Social Psychology, 101, 309-310.

Ramsden, P. (1988). Context and strategy: Situational influences on learning. In R. R. Schmeck (Ed.), Learning strategies and learning styles. Nova Iorque: Plenum Press.

Ratingan, B. (1989). Counseling in higher education. In W. Dryden, D. Charles-Edwards $\&$ R. Wolfe (Eds.), Handbook of counseling in Britain. Grã Bretanha: Routledge.

Rocco, M. T. F. (1981). Crise na linguagem: A redacção no vestibular. São Paulo: Mestre Jou.

Rosário, P. S. L. (1999). As abordagens dos alunos ao estudo: Diferentes modelos e suas interrelações. Psicologia: Teoria, Investigação e Prática, 1, 43-61.

Rose, H. A. \& Elton, C. F. (1971). Attrition and the vocationally undecided student. Journal of Vocational Behaviour, 1, 99-103.

Russell, R. K. \& Petrie, T. A., (1992). Academic adjustment of college students: Assessment and counseling. In S. D. Brown \& R. W. Lent (Eds.), Handbook of counseling psychology. (2. ${ }^{\mathrm{a}}$ ed.). Nova Iorque: John Wiley \& Sons.

Sanford, N. (1962). Developmental status of the entering freshman. In N. Sanford (Ed.), The American college: A psychological and social interpretation of the higher learning. Nova Iorque: John Wiley \& Sons.

Schlossberg, N. K. (1981). A model for analyzing human adaptation to transition. The Counseling Psychologist, 9(2), 2-18.

Schlossberg, N. K. (1998). Schlossberg's transition theory. In N. J. Evans, D. S. Forney \& F. G. Dibritto (Eds.), Student development in college: Theory, research, and practice. São Francisco: Jossey-Bass Higher and Adult Education Series.

Scwitzer, A. M., Ancis, J. R. \& Griffin, O. T. (1998). Validating a proposed model of african-american students'social adjustment. Journal of The First-Year Experience, 11, 1, 77-102.

Seligman, L. (1994). Developmental career counseling and assessment (2. ${ }^{\text {a }}$ ed.). Londres: Sage.

Soares, A. P. (1998). Desenvolvimento vocacional de jovens adultos: A exploração, a indecisão e o ajustamento vocacional em estudantes universitários, Dissertação de mestrado. Braga: Universidade do Minho.

Stern, R. A. (1986). Pride of place: Building the American dream. Boston: Houghton Mifflin.

Stone, G. L. \& Archer, J. (1990). College and university counseling centers in the 1990's: Challenges and limits. The Counseling Psychologist, 18, 539-607.

Strange, C. C. (1996). Dynamics of campus environments. In S. R. Komives, U. Delworth \& D. Woodard (Eds.), Student services: A handbook for the profession. São Francisco: Jossey-Bass Publishers.

Tavares, J., Santiago, R. A., Lencastre, L. \& Soares, I. (1996). Níveis de sucesso dos alunos do 1. ${ }^{\circ}$ ano dos cursos de Ciências e Engenharia da Universidade de Aveiro (relatório policopiado). Aveiro: Universidade de Aveiro.

Taveira, M. C. (1997). Exploração e desenvolvimento vocacional de jovens: Estudo sobre as relações entre a exploração, a identidade e a indecisão vocacional, Dissertação de Doutoramento. Braga: Universidade do Minho.

Tinto, V. (1987). Leaving college: Rethinking the causes and cures of student attrition. Chicago: University of Chicago Press. 
Traub, G. S. (1982). Relationship between locus of control and grade point average in freshmen college students. Psychological Reports, 50, 1294.

Upcraft, M. \& Gardner, J. (1989). The freshman year experience: Helping students survive and succeed in college. São Francisco: Jossey-Bass Publishers.

Weidman, J. (1989). Undergraduate socialization: A conceptual approach. In J. Smart (Ed.), Higher education: Handbook of theory and research (Vol. 5). Nova Iorque: Agathon.

Weinstein, C. S. (1979). The physical environment of the school: A review of the research. Review of Educational Research, 49, 577-610.

Wick, S. \& Shilkret, R. (1986). College adjustment and parental influence. South Hadley, MA: Mount Holyoke College.

Winston, R., Miller, T. \& Princo, J. (1987). Student Developmental Task and Lifestyle Inventory. Athens, GA: Student Development Associates.

College Adaptation and Transition: The Academic Life Experiences Questionnaire (abstract) The purpose of this paper is to analyze the process of transition and adaptation to the university of college students and to present the Academic Life Experiences Questionnaire (ALEQ; Almeida \& Ferreira, 1997). This questionnaire has been developed as a screening instrument and is conceptualized to describe the college student transition and adaptation as a dynamic process of interaction between the individual (with certain psychological and cognitive characteristics) and the academic and environmental demands. The ALEQ was administered to a sample of 1273 first-year students from different majors of Minho University. The results on the 17 scales of the instrument showed good psychometric properties. The internal consistency coefficients ranged from 0,69 to 0,88 . A factor analysis conducted revealed five factors explaining $72 \%$ of the variance. The five factors were designed as: (i) personal dimensions; (ii) adaptation to college major; (iii) context dimensions; (iv) academic learning process; and (v) family and financial management. 